\title{
Eco innovación y tecnología en el sector de la energía en la república dominicana ${ }^{1}$
}

\section{Eco Innovation and Technology in the Energy Industry in the Dominican Republic}

Ricardo-Joel Almánzar-Fortuna²

\section{Palabras clave:}

Economía, innovación, energía, sostenible, medio ambiente.

Artículo de investigación:

Fecha de recepción: 2019/09/05

Fecha de aceptación: 2019/12/16

Esta publicación se encuentra bajo licencia:

Creative Commons ReconocimientoNoComercialSinObraDerivada 4.0 Internacional

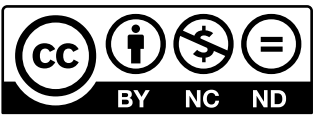

\section{Resumen}

La energía para el desarrollo y crecimiento de cualquier país juega un importante papel, en tanto que insumo de primera mano para lograr la transformación de bienes y servicios en el mundo actual. A partir de los hallazgos en la Cumbre de Río del 1992 el mundo se hizo eco de la necesidad de un cambio significativo en la forma de producir la energía, la cual, entre otros factores, influye de manera directa en la conservación del medio ambiente y consigo un arrastre de la calidad de vida y salud de las personas. En la década actual se ha logrado grandes avances en la producción energética de fuentes renovables y limpias, con lo que se persigue reducir la huella de carbono, la emisión de gases de efecto invernadero, además de tener un esquema de generación más eficiente y de menor costo a largo plazo, aportando de tal forma a una sostenibilidad en el tiempo y una sustentabilidad en el presente. Para los seres humanos el tener acceso a la energía repercute de forma directa e indirecta en su calidad de vida, pues se logra reevaluar el uso de los recursos naturales para las actividades cotidianas como el alumbrado,

Esta disertación es producto de una investigación continuada iniciada como requisito de aprobación del módulo de Economía Ambiental del programa doctoral de la UCI México, 2016; ésta fue publicada en su primera parte en la Revista científica Ágora Trujillo de la Universidad de los Andes, Venezuela, año 19 - Nº 38 Julio-diciembre 2016. Disponible en: http://www.saber.ula. ve/handle/123456789/43635

2 Doctor en Ciencias Económico Administrativas, República Dominicana, rj.almanzarf@gmail.com, código ORCID: https://orcid.org/0000-0003-2353-397X 


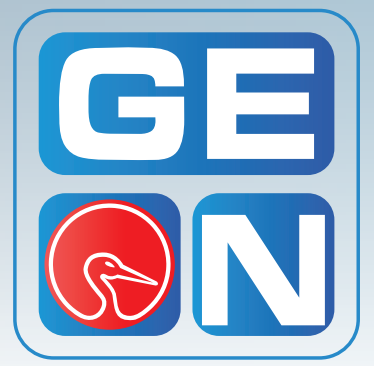

\section{Revista GEON}

(Gestión, Organizaciones y Negocios)

ISSN: 2346-3910 en línea

revistageon@unillanos.edu.co

Universidad de los Llanos

Colombia

\section{Almánzar Fortuna, R. (2020).}

Eco Innovación y tecnología en el Sector de la Energía en la República Dominicana.

\section{Revista GEON}

(Gestión, Organizaciones y Negocios), 7(1), 26-48.

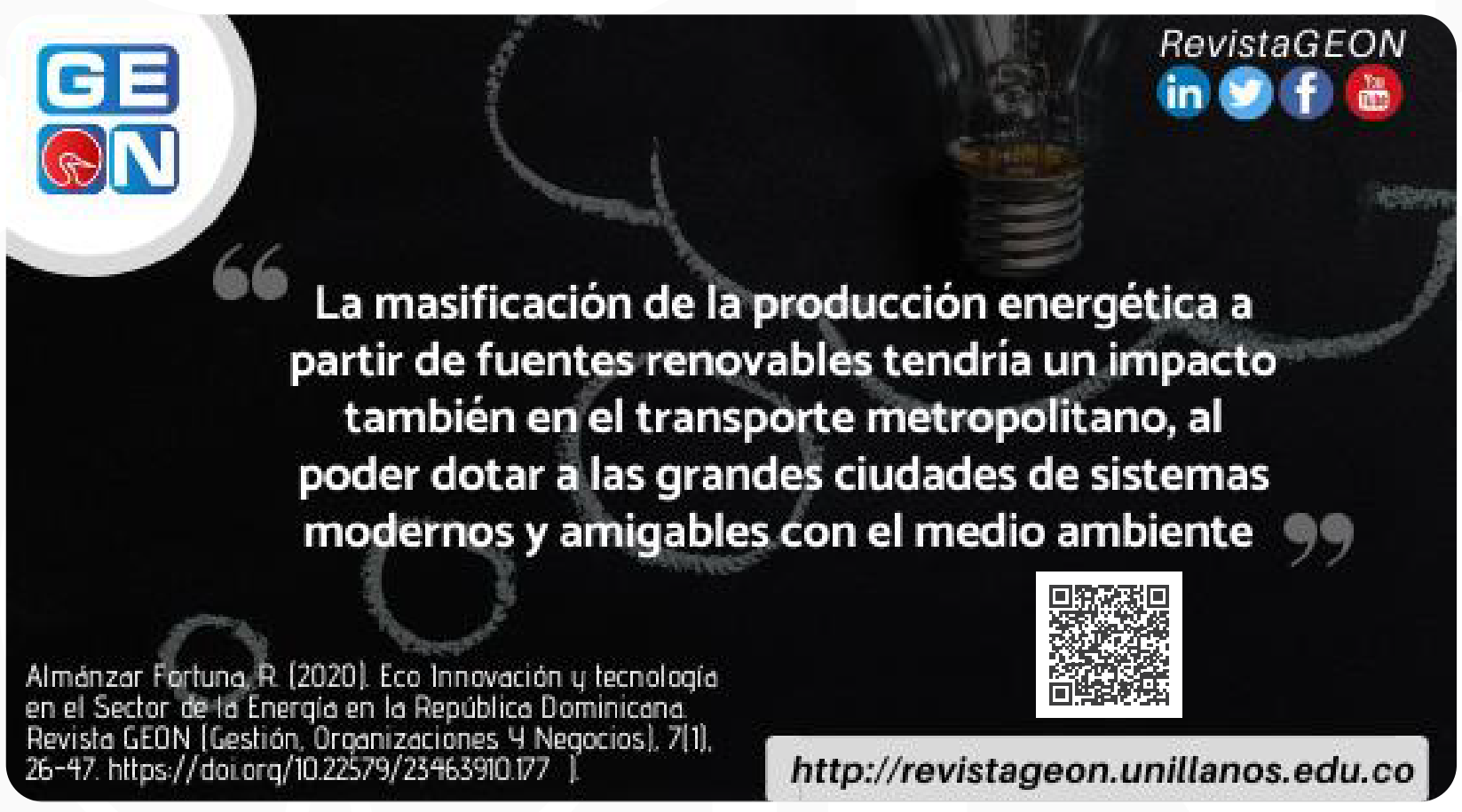


la cocción de los alimentos con leña, entre otros. Pero también a nivel general la gente se siente más cómoda y a gusto, a la misma vez que su nivel de resiliencia crece.

Palabras clave: Economía, innovación, energía, sostenible, medio ambiente.

\section{Abstract:}

The energy for the development and growth of any country plays an important role, while first-hand input to achieve the transformation of goods and services in the world today. Based on the findings in the 1992 Rio Summit the world echoed the need for a significant change in the way of producing energy, which among other factors, has a direct effect on the conservation of the natural environment and with a drag of the quality of life and health of the people. In the current decade has made great advances in the production of energy from clean, renewable sources, with the aim to reduce carbon footprint, the emission of greenhouse gases, in addition to having a schema generation more efficient and less expensive in the long term, giving way to a sustainability over time and a sustainability in the present. For human beings to have access to energy has a direct and indirect in their quality of life, as it is able to re-evaluate the use of natural resources for everyday activities such as lighting, cooking food with firewood, among others. In addition, on the general level people feel more comfortable and at ease, at the same time that their level of resilience grows.

Keywords: Economy, innovation, energy, sustainable, environment.

\section{Introducción}

La cumbre de Río del 1992 sentó las bases, por medio de la evidencia aportada por los científicos, para que se tomara en consideración tomar acciones drásticas que se encaminaran a la conservación de los recursos naturales y medio ambientales del planeta. Ello debido a la toma de datos que reflejó un cambio en el comportamiento climático. Pero además 
se evidenció que el papel humano era de vital importancia para minimizar los efectos del fenómeno. Es entonces cuando se toma nota de que las emisiones de dióxido de carbono y el efecto de los gases invernaderos perjudican al medio ambiente, a las personas y también es potenciador de las reacciones del planeta ante dicho cambio.

Uno de los ejes a trabajar es la producción energética, la cual involucra grandes esfuerzos de los gobiernos, empresas y las personas, dado que es una labor conjunta el lograr el desprendimiento del uso de los combustibles fósiles para la generación eléctrica a gran escala, o por lo menos en un papel protagónico. Por lo que los países de del G20 se tomaron en serio el asunto y empezaron la toma de acciones en lo referente a este y otros temas.

Es finalmente en la Agenda 2030 de la Organización de las Naciones Unidas, ONU, en donde se declara, junto con otros temas, la importancia que tiene la energía para el desarrollo y crecimiento de las personas y las naciones, pero también se delinea que esta debe provenir de fuentes amigables al medio ambiente para que se eficiente y efectiva. Es de tal forma como se empieza la transición hacia la producción energética por medio del sol, agua, viento y otras, en los países más desarrollados que están dentro de la Organización para la Cooperación y Desarrollo Económico, OECD.

La República Dominicana como firmante de los acuerdos internacionales que buscan reducir los efectos del cambio climático, se ve en la necesidad de dar cumplimiento a los mismos. Para ello debe transformar y diversificar su matriz energética con miras a tener una energía limpia, accesible para la gente y que conserve el medio ambiente. Sin embargo, los retos son muchos debido a la mala administración histórica que ha tenido el sector en los últimos cincuenta años, cono pocas mejoras significativas, en donde el factor político es crucial. La voluntad política es un factor definitivo y depende de la propia conducta personal de aquellos que gobiernan y deciden los destinos de una nación, lo que conlleva a que la toma de decisiones sea fundamental para el desarrollo y crecimiento de las comunidades que integran un Estado. La administración de los recursos de la sociedad es importante porque los recursos son escasos. (Almánzar, 2016)

La investigación realizada presenta evidencia relevante que enlaza de forma crítica el papel del Estado como ente estimulador del desarrollo y crecimiento, con la necesidad de las personas de una garantía de acceso a los servicios básicos, como salud, educación y la energía, misma que es un insumo de primera necesidad para la realización de las actividades diarias de todo ser humano, y de las empresas en la transformación y comercialización de bienes y servicios, todo en el contexto de un mundo moderno y que crece exponencialmente en número y tecnologías.

\section{(i) Organización y métodos}

El tipo de estudio tiene un enfoque mixto, en el que se combinan las herramientas cualitativas y cuantitativas para logar una recopilación y análisis de 
datos que aporten fiabilidad a la misma, así como un esquema de análisis por objetivo que garantice la ejecución de la misma dentro del tiempo pactado.

Según los estudios teóricos, la investigación corresponde a un modelo no experimental, dado que no existe la posibilidad de manipular variables. Por otra parte, la posibilidad de analizar el fenómeno a través de lo largo del tiempo lo convierte en uno de tipo longitudinal, por la posibilidad de analizar tendencias. Una última clasificatoria lo convierte en transeccional o transversal, en tanto se estudiará una correlación de variables. (Hernández, Fernández y Sampieri, 2010).

Se aplicó una encuesta dentro de los límites de la Provincia San Juan, Región del Valle, al sur República Dominicana. El objetivo era conocer la opinión de los usuarios del servicio eléctrico y si se puede establecer al- guna relación entre el acceso a este servicio y el desarrollo de las personas. Se realizó a un total de 422 hogares elegidos al azar, por medio de un muestreo aleatorio simple, con un método de elección de 5 por 1.

La muestra seleccionada se obtuvo a partir de los datos del Censo de población y vivienda del 2010, en el que se contabilizó un total de habitantes de 232,333 en la provincia San Juan, con una distribución de 72,358 viviendas, de las que se debió encuestar 383, según la aplicación de la fórmula con intervalo de confianza de 95\% y error de 5\%.

Se utilizó además entrevistas a grupos de usuarios, grupo de expertos en distintos ámbitos profesionales, investigación bibliográfica hasta los avances más recientes en energía sostenible y análisis de tendencias de crecimiento de consumo y población.

Tabla 1: Herramientas y procedimientos de la investigación

\begin{tabular}{|c|c|}
\hline Objetivo General & Herramienta \\
\hline $\begin{array}{l}\text { Determinar qué efectos tiene la eco innovación en la industria de la } \\
\text { energía en la República Dominicana }\end{array}$ & $\begin{array}{l}\text { - Entrevistas a grupos } \\
\text { - Entrevistas a expertos } \\
\text { - Investigación bibliográfica } \\
\text { - Encuestas }\end{array}$ \\
\hline Objetivos específicos & Herramienta \\
\hline $\begin{array}{l}\text { Analizar si el uso de las energías limpias influye en la conservación } \\
\text { del medio ambiente }\end{array}$ & $\begin{array}{l}\text { - Investigación bibliográfica } \\
\text { - Proyección cuantitativa } \\
\text { - Entrevistas a expertos }\end{array}$ \\
\hline $\begin{array}{l}\text { Determinar si la implementación de energías limpias tiene un } \\
\text { impacto en el bienestar socio económico y cultural delas personas }\end{array}$ & $\begin{array}{l}\text { - Investigación bibliográfica } \\
\text { - Entrevistas a grupos }\end{array}$ \\
\hline $\begin{array}{l}\text { Determinar si el modelo innovación de energías limpias impacta en } \\
\text { el presupuesto de inversiones del Estado }\end{array}$ & - Análisis de tendencias(Trend) \\
\hline $\begin{array}{l}\text { Establecer si contribuiría la eco innovación en la energía al desarro- } \\
\text { llo sostenible del país }\end{array}$ & $\begin{array}{l}\text { - Entrevistas a expertos } \\
\text { - Análisis de tendencias (Trend) } \\
\text { - Encuestas }\end{array}$ \\
\hline
\end{tabular}

Nota: Se utilizó un enfoque mixto para desarrollar cada objetivo, basado en los aportes y lineamientos de Hernández, R., Fernández, C. y M. Sampieri. (2010).

Fuente: elaboración propia 
Tabla 2: Datos de los encuestados

\begin{tabular}{cccccc} 
& Edades & & \multicolumn{3}{c}{ Sexo } \\
$18-25$ & 128 & $31.8 \%$ & Femenino & 242 & $57.3 \%$ \\
$26-35$ & 133 & $33.1 \%$ & Masculino & 180 & $42.7 \%$ \\
$36-45$ & 85 & $21.1 \%$ & & \\
$45+$ & 56 & $13.9 \%$ & & \\
No tipificado & 20 & $4.7 \%$ & & \\
\hline
\end{tabular}

Nota: Muestra seleccionada en la Provincia San Juan con base en el último Censo poblacional del 2010.

Fuente: elaboración propia

\section{(ii) Revisión de literatura}

\section{La energía renovable y las personas}

La importancia de la energía radica en su potencial transformador del esfuerzo humano, ya sea nivel personal, familiar, comunitario o industrial. Ésta se puede utilizar para el cultivo de frutos y víveres, labores agrícolas diversas, en tanto que se constituye en un insumo que interviene en el proceso productivo, así tanto en bienes y servicios.

En la actualidad resulta contraproducente para cualquier ser humano el carecer del acceso a la energía, ya sea esta para iluminarse en las noches, conservar alimentos o también emplearla en labores de riego de terrenos. Pero también las empresas la necesitan para mantener las distintas maquinarias operando.

En tal sentido, según aporta la ONU (2014):

La energía sostenible es un factor clave del desarrollo sostenible para todos los países y todos los pueblos. La energía es esencial para aliviar la pobreza, mejorar el bienestar humano y elevar el nivel de vida. La energía ha sido una preocupación central para la humanidad a lo largo de su historia. Además, los servicios energéticos fiables son una condición previa para las inversiones que propicien el crecimiento económico y el desarrollo social. La disponibilidad de servicios energéticos es un fuerte factor determinante de las inversiones en los sectores industrial, transporte, servicio y todos los demás sectores principales de la economía de un país. Los servicios de energía modernos permiten también importantes mejoras en la educación y la salud. Estos son dos factores fundamentales en el desarrollo del capital humano. La disponibilidad de energía sostenible promueve la equidad y aumenta las oportunidades facilitando el empoderamiento y la mejora del bienestar de la población.". (p. 1)

El uso de las energías limpias sirve de plataforma para el impulso económico de los países que desean hacer la transición a una producción amigable con el medio ambiente. En tal sentido, Boztepe y Karaca (2009), (citados por Sari y Akkaya 2016), aportan que "la biomasa contribuye a la economía 
rural y nacional mediante la creación de oportunidades de empleo más que otros sectores energéticos y estimular la economía y la reducción de la importación. La biomasa es una solución ecológica". (p. 322) Lo que sucede es que los agricultores pueden cultivar para vender biocombustibles a las empresas para la generación eléctrica. Asimismo, su calidad de vida mejora debido a los diversos usos que le pueden dar a dichos combustibles.

Dentro de las ocupaciones que se verán afectadas positivamente con el crecimiento de las energías renovables están los gerentes de proyectos, ingenieros eléctricos, analistas de datos, mecánicos o constructores, técnicos de servicios, educadores, entre otros. Asimismo, hay efectos positivos de las instituciones en la economía de los países, los cambios económicos de las pequeñas, medianas y grandes empresas e instituciones en los países industriales y los beneficios de los países para su desarrollo como consecuencia de una mejor y más eficiente producción. (Boztepe y Karaca, 2009)

Por otra parte, el Estado es un interventor que juega un rol central en la planeación del desarrollo y crecimiento de las personas, en tanto que como declarador de políticas deberá estimular a que se realicen proyectos de innovación tecnológica que beneficie a las personas y que a la vez faciliten a las empresas embarcarse en nuevos modelos productivos.

Según aporta Bossink (2017):

La revisión de la literatura concluye también que muchos de los resul- tados de los proyectos de demostración se utilizan para aprender a desarrollar la política pública que estimula el uso de formas de energía sostenibles. A nivel internacional, la Unión Europea, por ejemplo, desarrolla una política, reglamentación y legislación para exigir que los logros de los proyectos de demostración son utilizados por otros actores, fuera de los proyectos de demostración. Otro ejemplo nacional es Finlandia, donde el gobierno utiliza a los proyectos de demostración para aprender a desarrollar mejores políticas de innovación sostenible.

Un ejemplo ilustrativo es el programa nacional de construcción de proyectos de demostración de eficiencia energética en los Países Bajos, en la década de los noventa. Las autoridades utilizaron los resultados, experiencias, conocimientos y mejores prácticas del programa en el que se ejecutaron diversos proyectos de demostración para afinar las normas nacionales en materia de eficiencia energética de todos los regulares, a construirse nuevos edificios en el país. Estas normas fueron parte integrante de la ley, y agudizado en dos a tres años. (p. 9)

Es por los anteriores ejemplos que se puede llegar al argumento de que el gobierno central es la clave mayor para que las implementaciones de los proyectos de energía renovable surjan el efecto esperado en el bienestar humano y medio ambiental, y en última instancia que los inversores puedan recuperar el capital dispuesto 
para tales iniciativas. Sin embargo, es evidente también que la percepción pública es uno de los factores que detractan el curso de la transición hacia una energía realmente sostenible.

De acuerdo a los aportes de Botelho, Pinto, Lourenço, Valente y Sousa (2016):

No hay ninguna duda acerca de la complejidad alrededor de la aceptación social de las innovaciones en energía renovable. Según Wüstenhagen et al. (20) El concepto de aceptación social de las innovaciones en energía renovable es multidimensional, incluida la aceptación socio-político, la aceptación de la comunidad y la aceptación del mercado. Aceptación socio-política es "la aceptación social en el más amplio, nivel general" (20, p. 2684). Se refiere al papel de los ciudadanos. Principalmente se manifiesta a través de un apoyo general a una tecnología basada en renovables o políticas para apoyar su desarrollo. Esto a menudo se mide a través de encuestas de opinión que representan los individuos' actitudes agregados $(23,31,32)$. Aceptación sociopolítica conductora ayuda a establecer condiciones para la realización de innovaciones. Se trata de la disposición de los actores (público en general, los principales interesados y responsables políticos) para generar cambios institucionales y políticas que creen condiciones favorables para las nuevas tecnologías (33).

La aceptación de la comunidad se refiere al papel de los consumido- res como voluntaria o involuntaria de los usuarios de la tecnología. Desempeña un papel importante en los casos en que la adopción de una innovación afecta a grupos de agentes, como las tertulias de decisiones para las instalaciones de energía renovables (23). Un enfoque comunitario eficaz es esencial para la implementación de las energías renovables. Los estudios realizados sobre este tema muestran que algunos de los factores que parecen ser esenciales para el éxito de proyectos de energía renovable, tales como la colaboración en la toma de decisiones, empleando formas eficaces de participación de la comunidad; la participación efectiva de la comunidad en el área de proceso o en la gestión y la propiedad, que permite a la comunidad se identifiquen con el proyecto; la percepción de cómo el nuevo sistema se adapta a la identidad de la comunidad; el hecho de que el proceso de toma de decisiones es percibido como justo; y la existencia de una confianza mutua entre los miembros de la comunidad y los inversores y propietarios de la infraestructura (33-36). (p. 75-76)

Según se expresa en el programa de desarrollo de la ONU (2011):

Los pobres están en situación particularmente desventajosa. Los pobres de las zonas urbanas suelen tener algún acceso a la electricidad, pero su calidad es deficiente, el servicio no es confiable y es intermitente, y sus conexiones a menudo son informales. No ser clientes regula- 
res y no pagar las tarifas normales que otros clientes hacen, su acceso a la electricidad puede resultar caro. Tienen que confiar en la búsqueda de rentas a intermediarios para permanecer conectado. En las zonas rurales, el acceso físico es a menudo inexistente. Si los pobres de las zonas rurales no tienen acceso a la electricidad, tiende a ser de mala calidad y/o cantidad de sistemas independientes o mal gestionado y mini redes ineficientes que son costosas y propensas a fallos frecuentes.

Los países en desarrollo, en particular, la necesidad de ampliar el acceso a fuentes fiables y modernos de energía a fin de reducir la pobreza y mejorar la salud de sus ciudadanos, al mismo tiempo, promover el crecimiento económico y la mitigación del cambio climático. Invertir en limpias, eficientes, asequibles y fiables sistemas de energía es indispensable para un futuro próspero, ambientalmente sostenible. Asegurar la seguridad energética requerirá la diversificación de tipos y fuentes de energía, con un creciente enfoque en las necesidades de los consumidores, sobre poblaciones de los suministros de energía, la eficiencia energética y la interconexión regional.

Haciendo la energía moderna y accesible sobre una base sostenible para los pobres pueden tener los siguientes beneficios:

- Reducir la pobreza y crear empleos, en particular para los hogares pobres y emprendedores, chispa de generación de ingresos, reducir el hambre y aumentar la productividad agrícola y las oportunidades de negocio.

- Potenciar a la mujer mediante la liberación de las mujeres y las niñas de las tareas que consumen mucho tiempo, liberando tiempo para la educación y la actividad económica.

- Mejorar las condiciones de salud de las mujeres y los niños por la eliminación de la "cocina" de humo.

- Promover soluciones de energía limpia que puede contribuir a un bajo contenido de carbono y el cambio climático futuro resistente.

Dada la importancia de la energía sostenible para lograr el desarrollo humano, el PNUD ha hecho de la prestación de servicios sostenibles de energía, especialmente a los pobres, una prioridad. Su trabajo sobre la energía sosteniblees guiado por la energía-desarrollo vínculos identificados anteriormente. Con oficinas en 135 países, el PNUD está en una posición única para ayudar a los países en desarrollo a abordar las cuestiones relativas a la energía. (p. 2-3)

Asimismo, la transformación del uso de energía tiene diversas vertientes, ya no se habla simplemente de consumirla, sino que hacerlo de una manera responsable y eficiente. Por esta razón la educación de las personas y empresas como consumidores es un tópico de gran importancia para logar una transición hacia el esquema sostenible. 
Según aporta la Agencia Internacional de Energía, IEA, 2015:

La política de eficiencia energética está evolucionando desde una inocente concepción de "ahorro de energía" hacia una que busca cada vez más revelar los resultados de la diversidad de consumidores y sociedades, para lograr una mejor comprensión. Esto incluye por ejemplo la posibilidad de brindar mejores servicios sociales, de salud y abordar las desigualdades sociales en el sector residencial, así como los desafíos que surgen en un ambiente de austeridad como consecuencia de la crisis financiera global. (p. 24)

Las necesidades humanas de abrigo, servicio de alimentación, higiene, movilidad y entretenimiento impulsan el uso de tecnologías que se encuentran en el núcleo de la demanda energética del sector residencial. Las modernas tecnologías consumidoras de energía continúan transformando los hogares con servicios progresivamente más eficaces, liberando a los ocupantes de las tareas manuales asociadas al lavado de la ropa y de la vajilla; permitiendo un almacenamiento y cocina de alimentos más seguro, mejorando el confort y la iluminación; y abriendo cada vez más nuestros hogares a un mundo de educación visual y auditiva, así como a recursos de entretenimiento. (p. 55)

El principal factor que afecta al consumo energético en el sector servicios es el nivel de actividad económica, que suele ser representado por el valor añadido resultante del sector. Niveles más altos de actividad económica inducen a un incremento en la actividad comercial y en el parque de viviendas y edificios, y a un mayor número de empleados en el sector. Ambos efectos llevan a un incremento en la demanda de servicios energéticos. Las tendencias en el consumo de energía final total también son influenciadas por el clima, la superficie edificada, el tipo de edificio (relativo al sector de actividad), la antigüedad de los edificios, la madurez de una cierta economía, la calidad de gestión energética del edificio, el ingreso per cápita, las condiciones climáticas y las mejoras en la eficiencia energética. El perfil económico y demográfico también tiene un impacto en la estructura del sector. (p. 59)

La energía es un factor clave para todos los productos manufacturados. Manteniendo todos los otros parámetros constantes, un aumento en la producción industrial generalmente dará lugar a un aumento en el consumo energético; dado que la mayoría de los procesos tienen componentes fijos y variables en su funcionamiento, cuando la demanda de productos de un cierto proceso disminuye, la intensidad energética aumentará. Esta relación entre la energía y la producción, y el modo en que compara distintos países, estará influenciada por varios factores, como por ejemplo: la antigüedad promedio de las plantas (las plantas nuevas o reconstruidas son 
por lo general más eficientes que otras más antiguas), las prácticas de mantenimiento, la calidad de la energía utilizada (p.ej.: el valor calorífico); la calidad del producto manufacturado, las materias primas utilizadas, la calidad requerida del producto (p.ej.: el nivel de pureza), el proceso o tecnología utilizada; y, a un nivel más agregado, la composición del sector industrial. (p. 86)

En su conjunto, todos los argumentos anteriores tienen como resultante un efecto significativo en el bienestar de las personas, pues si se incrementa la demanda de la energía para usos productivos las empresas tienen que aumentar la producción y ello puede generar más empleo. Si se logra establecer que esa producción es más efectiva por medio de fuentes energéticas renovables, entonces se tiene un grado de sostenibilidad, mayor.

\section{La energía renovable y el país}

Los beneficios de la implementación de energías limpias afectan a las personas de forma directa, pero también se puede establecer que el país como tal también gozaría de ventajas macro a largo plazo, pues en el entendido de que la inversión inicial para el despliegue de proyectos a gran magnitud y a lo largo de toda la geografía nacional es cuantiosa, bien se puede recuperar el capital a través del tiempo a la misma vez que se palean los efectos de los gases de efecto invernadero y el cambio climático en general.

Según la investigación de Ibrahiem (2015):
Un número de estudios empíricos han investigado la relación entre crecimiento económico y consumo de energía renovable o consumo de electricidad renovable, ya sea dentro del país o contexto estudios con datos de panel. A partir de esos estudios dentro del contexto de cada país, Sari et al. (2008) examinaron la relación entre el consumo de energía desagregar (carbón, combustibles fósiles, energía hidroeléctrica, energía solar, energía eólica, madera y residuos) y la producción industrial y el empleo en EE.UU. utilizando el modelo ARDL y concluyó la presencia de co-integración entre las variables. Yildirim et al. (2012), aplicado Toda-Yamamoto procedimiento bootstrap y test de causalidad corregido para los datos de los Estados Unidos, y encontraron la hipótesis de crecimiento entre la biomasa de residuos y el consumo de energía del crecimiento económico, y no se encontró relación de causalidad entre el crecimiento económico y otros tipos de energía renovable (biomasa, consumo de energía Consumo de energía hidroeléctrica, energía geotérmica, el consumo, el consumo total de energía de la biomasa y el consumo de energía derivada de la madera). Pao y Fu (2013) investigaron la relación entre crecimiento económico y diversos tipos de consumo de energía en Brasil y encontró resultados mixtos como hipótesis de conservación entre el consumo de energía no renovable y el crecimiento económico, la hipótesis de crecimiento entre no-renovables hidroeléctrica el consumo de 
energía y el crecimiento económico y la hipótesis de retroalimentación entre el crecimiento económico y el consumo total de energía renovable. (p. 316)

Además del análisis de un solo país, existen estudios con datos de panel, comenzando con Sadorsky (2009), que examinó la relación entre el ingreso y el consumo de energía renovable para las economías emergentes, y mediante la aplicación de instrumentos de co-integración concluyó que el ingreso per cápita real afecta positivamente el consumo per cápita de energía renovable. Apergis y Payne (2011) examinaron la relación entre consumo de energía renovable y no renovable y el crecimiento económico en los países desarrollados y los países en desarrollo y encontrar la causalidad bidireccional entre las variables tanto de corto y largo plazo. Asimismo, Apergis y Payne (2012), aplicado instrumentos modelo de corrección de errores e investigó la relación de causalidad a la Granger entre crecimiento económico y renovables y no renovables, el consumo de electricidad para América Central de datos, y encontró que la relación entre el consumo de energía renovable y el crecimiento económico confirma la hipótesis de crecimiento económico en el corto plazo y comentarios hipótesis a largo plazo, y también encontraron la causalidad bidireccional entre el consumo de electricidad no renovable y el crecimiento económico en ambos a corto y largo plazo.
A partir de estudios anteriores, puede concluirse que se encontraron resultados mixtos para el crecimiento económico del consumo de energía renovable nexus o el crecimiento económico- el consumo de electricidad renovable y Sebri nexus (2015) en su meta-análisis de consumo de energía renovable-crecimiento económico nexus concluyó que la variación en los resultados puede atribuirse a varias características como las técnicas de estimación, Especificación de modelo, las características de los datos y el nivel de desarrollo del país. (p. 317)

Las fuentes de energía juegan un papel crítico en el desarrollo, especialmente en los países en desarrollo en general y en Egipto en concreto, pero ahora Egipto está luchando para satisfacer las necesidades de energía a pesar de que era exportador neto de petróleo en el pasado, así que es muy esencial para cambiar a otra fuente de energía esencialmente renovable recursos energéticos que son vastos y fuente limpia. Por lo tanto, el objetivo de este estudio consiste en examinar la relación entre crecimiento económico, consumo de electricidad renovable y la inversión extranjera directa. Esto se realiza mediante la realización de ARDL modelo y test de causalidad de Granger entre crecimiento económico y consumo de electricidad renovable, por un lado, y el crecimiento económico y la inversión extranjera directa en el otro lado. El estudio confirma la presencia de co-integración entre crecimiento 
económico, consumo de electricidad renovable y la inversión extranjera directa y que tanto el consumo de electricidad renovable y la inversión extranjera directa se correlacionan positivamente con el crecimiento económico. (p. 321)

A pesar de que las energías renovables representan una oportunidad de mejora a nivel macro, existen obstáculos y detractores para este tipo de proyectos, por lo que el gobierno tiene que garantizar que la ejecución e implementación no se vea afectada por fuerzas exógenas a los mismos. Es este marco en donde se deberá proveer al aparato de productivo de los incentivos necesarios y exenciones fiscales que sirvan como un medio para la continuidad del plan de acciones a largo plazo.

Según lo hallado por Shukla, Sudhakar, y Baredar (2017):

A pesar del enorme potencial y las ventajas de la promoción de las energías renovables, hay obstáculos tanto en los niveles nacional y regional, que deben ser superados. Uno de los desafíos es la baja inversión en energía renovable, porque implica altos costos de capital inicial y los beneficios monetarios derivados de tales proyectos se toman tiempo para materializarse. (p. 3)

En contraposición a la implementación de los modelos de energía renovable se encuentran los diversos retos mencionados anteriormente, mismos que obedecen a razones financieras, gubernamentales y de conocimiento, es decir de educación de la población general. Crear la conciencia macro y que todos los sectores de la economía nacional estén informados del porqué se debe hacer la transición hacia un esquema renovable no es fácil, pero ni no se toman acciones ahora, luego serán más grandes las consecuencias.

El reporte del Organismo Internacional de Energía Atómica, OIEA, (2008):

Durante el siglo XX, la temperatura media de la superficie de la tierra se elevó alrededor de 0,6 ${ }^{\circ} \mathrm{C}$, y abundan cada vez más las pruebas de que la mayor parte de este calentamiento es imputable a las concentraciones crecientes de GEl en la atmósfera. Por ejemplo, la cantidad de $\mathrm{CO} 2$ se ha incrementado en más del 30\% desde la época preindustrial y actualmente está aumentando a una tasa sin precedentes de 0,4\% por año, debido sobre todo a la combustión de combustibles fósiles y a la deforestación. Las concentraciones de CH4y N2O están subiendo también a causa de las actividades energéticas, agrícolas, industriales y de otra índole. Las concentraciones de monóxido de nitrógeno (NO), dióxido de nitrógeno (NO2), monóxido de carbono (CO) y compuestos orgánicos volátiles no-metánicos (COVNM) también están en alza como resultado de la actividad antropógena. Aunque estos gases no son por si mismos de efecto invernadero, afectan a la composición química de la atmósfera, lo que redunda en un incremento del ozono troposférico, que es un gas de efecto invernadero (GEI). 
Según las predicciones, los efectos resultantes darán lugar a repercusiones climatológicas más extremas que en el pasado, con zonas que experimentarán muchas más tormentas y lluvias, mientras que otras sufrirán sequías. Aún no se sabe a ciencia cierta con qué rapidez ni dónde se producirá ese cambio, pero las consecuencias podrían ser graves, especialmente en los países en desarrollo, que son los que están en peor situación para prepararse y luchar contra los efectos de condiciones meteorológicas extremas, como inundaciones, corrimientos de tierras, sequías, etc. (p. 101-102)

De acuerdo al reporte de Greenpeace (2014):

En particular, las políticas de fomento de las energías renovables pueden repercutir tanto positiva como negativamente en el gasto energético delos hogares por tres vías diferenciadas: en primer lugar, a través del cambio en los precios mayoristas, provocados por un aumento o disminución de los costes de producción; en segundo lugar, por cambios en los precios minoristas, debido a las variaciones de los costes de suministro $y$, finalmente, por medio de la alteración en el consumo de la energía de los hogares, derivada de cambios de comportamiento de los mismos o bien porque la prestación de determinados servicios energéticos se ajuste a medidas de eficiencia energética. A partir del análisis de la literatura existente, como podrá comprobarse en líneas posteriores, se puede señalar que existe un gran consenso académico y empírico sobre la relación directa entre la introducción de renovables y la disminución de los precios mayoristas de la energía.

\section{(iii) Resultados y análisis}

En lo que tiene que ver con la experiencia usando energía eléctrica existe la percepción predominante de un servicio que es regular frente a las expectativas de los actuales tiempos, debido a que el uso que se le puede dar y la capacidad instalada no van a la par, en contraposición se encuentra la expectativa de los usuarios que visualizan los beneficios de tener una energía constante. Las personas relacionan el desenvolvimiento diario, el desarrollo de sus labores cotidianas a la existencia del servicio de energía eléctrica; más aún, que en los tiempos corrientes es impensable pensar en alguna clase de progreso personal sin acceso a la energía. La modernidad se trata de poder realizar tareas cada vez más rápido, ya sea los quehaceres domésticos, ver el noticiario, leer el periódico; todo esto se logra optimizar en relación a la continuidad energética, misma sin la que es más difícil realizar los oficios más simples.

Es interesante que los precios sean un punto clave que distinguen las personas al hablar de energía en República Dominicana, la cual atribuyen a deficiencias operativas y a una tendencia local histórica. Existe poca información sobre los avances en el campo de las energías renovables, no siendo así el conocimiento de 
sus beneficios en relación al cuidado medioambiental. La relación servicio-optimización se percibe en consecuencia de una provisión mucho más estable y accesible en cuanto a sus precios, a la misma vez que influye de manera positiva en la conservación del medio ambiente.

Existe un marco de conciencia que se refiere principalmente el efecto contaminante de los gases de efecto invernadero por las elevadas emisiones de los mismos, haciendo hincapié de forma directa en que parte de la solución es cambiar a una producción más amigable con el medio ambiente y la naturaleza. Se exige del gobierno que sea un actor principal, un impulsor del cuidado al medio ambiente y propulsor de iniciativas de producción alternativa que sean viables y sostenibles. No existe negación en relación con la necesidad de cuidar el medio ambiente y mover los sistemas de producción a otros más eficientes y efectivos que los actuales. La importancia de un medio ambiente y naturaleza saludable influye en la sostenibilidad y sustentabilidad, haciendo énfasis en que las acciones deben tomarse desde ya para lograr palear la situación actual que es fruto de los efectos del cambio climático en sus distintas vertientes.

Por otra parte, limitado el acceso a la energía en muchas de las zonas rurales, en las que todavía se encuentran algunas sin el servicio y otros muy limitados. Las alternativas son escazas para contrarrestar la ausencia del servicio o las deficiencias en el mismo. La importancia que le dan las personas a la energía es mayúscula debido a que las exigencias actuales del hogar y para la vida cotidiana hacen indispensable tener acceso al servicio u alguna fuente alternativa. Las personas están conscientes de que es un insumo que no puede faltar y que debería estar presente independientemente de la zona geográfica. Las personas sienten la energía como una necesidad y su escasez se refleja en su calidad de vida, ya que la energía representa un estatus de confort y seguridad, un resguardo y abrigo para las personas.

Las personas conocen de la existencia de la energía solar e incluso han tenido acceso anteriormente, pero se dejó de dar seguimiento y mantenimiento del servicio. Hacen énfasis en que el papel del gobierno debe ser de gestor en el mejoramiento del servicio, por consiguiente, en una mejoría de la calidad de la calidad de vida. Aunque con limitaciones expresivas y falta de conocimiento por la falta de información, las personas entienden la diferencia entre una energía producida a partir de recursos renovables frente a otra que depende de combustibles fósiles. No se halla una opinión común, pero, subyace la necesidad de tener alguna forma del servicio, por lo que cada persona se adhiere a sus propias posibilidades, ya sea la migración, la contratación de un servicio caro, etc.

A su vez, si bien no existe una unanimidad de las opiniones, no es menos cierto en que la exposición más generalizada es que a lo largo de la historia el sector energético dominicano ha sido deficiente, debido a carencias de diversos tipos que van 
desde una administración ineficaz, producción insuficiente, hasta pérdidas por la instalación y deterioro de las redes que suplen el servicio. Es de notar que han existido esfuerzos a lo largo de los distintos gobiernos de turno, aunque no se ha priorizado la relevancia del sector como parte de la maquinaria productiva sino hasta el presente en donde se pretende solucionar el problema energético con la construcción de una planta a carbón, Punta Catalina. El papel que juega el Estado se percibe como el pilar central para poder tener un avance real y significativo en el sector energético, mismo que se ha tratado de impulsar en el pasado, pero por falta de manejo administrativo, así como la corrupción, no se ha logrado los objetivos a corto ni largo plazo, dando como resultante que los apagones sean un elemento constante de la vida cotidiana de los dominicanos, aunque que algunas mejorías en la última década.

Se tiene una conciencia en cuanto a la importancia del cuidado al medio ambiente y de los beneficios que una producción limpia conlleva para el bienestar de la gente y además para la conservación de los recursos naturales, que en el país si bien son abundantes, están siendo depredados y consumidos sin reposición oportuna, como el caso de la tala de árboles para producir carbón. Según el juicio de los expertos y profesionales de distintas áreas, el país cuenta con los principales recursos para una producción limpia y sostenible en el tiempo. Es notable que exista un reconocimiento de los esfuerzos actuales, que, aunque insuficientes, vienen a aportar a la producción total ener- gética de la república, en tanto que parques eólicos, hidroeléctricos y paneleros solares están cobrando cada vez más importancia.

La vertiente apunta a una necesidad de la inversión productiva eficiente, en relación a la relevancia de los costos que se pueden ver significativamente reducidos a largo plazos y, que en contraposición con el esquema actual en el que el Estado dominicano subsidia a las empresas generadoras de electricidad, tiene la ventaja principal de que son recursos renovables los que se usan y reúsan, además de que se puede cuidar la salud medio ambiental. Se puede afirmar que la energía, tal y como se ha establecido en la agenda de desarrollo del milenio por la Organización de las Naciones Unidas, ONU, es un ítem de importancia mayúscula para el impulso de la cotidianidad de las personas, el cual afecta su capacidad doméstica, su desenvolvimiento productivo. Pero, además, ayuda a que las personas tengan un mayor nivel de resiliencia y, de manera directa se puede traducir en bienestar personal el hecho de que los niveles de producción y transformación de bienes y servicios se ve positivamente afectada.

El papel del Estado debe ser de un impulsor, dado que carece de la disponibilidad de fondos suficientes, promover alianzas público-privadas cobra bastante sentido. Pero también se debe denotar que, si bien estos inversores privados cuentan con infraestructuras, normas, políticas y procedimientos establecidos, la ética gubernamental deja una brecha abierta hacia la corrupción o mala administración de los fondos y bene- 
ficios colaterales, cuestión que debe ser reformulada entonces en aras de garantizar una verdadera alianza y no una dependencia, un parasitismo del Estado. Éste puede mantener un empuje adicional al desarrollo de proyectos de energías limpias, toda vez que logre mantener el estímulo correcto con base en incentivos y exenciones fiscales que logren motivar a los distintos inversores a encaminarse en este tipo de modelo productivo, mismo que es caro al inicio pero que luego rinde sus frutos.

Para que el país dominicano pueda realizar la sustitución de la producción energética y moverse a un esquema de eco innovación, debe necesariamente ir transformando su engranaje institucional, estructural y además crear políticas y normas adicionales que garanticen transparencia en el manejo de sus fondos. El Estado debe realizar una planificación concreta que contemple entre sus objetivos la disminución de pérdidas en las redes, la transformación de la matriz energética, pero también crear políticas que obliguen a la continuidad de los planes a largo plazo, factor que en los países en vías de desarrollo es uno de los principales por los que los proyectos no se efectúan al tiempo estipulado. Así mismo, la educación de los funcionarios es vital, también la de la población en general, ya que es un esfuerzo que requiere de la cooperación de todos los ciudadanos.

\section{(iv) Conclusiones y recomendaciones}

Los objetivos de desarrollo del milenio de la ONU engloban un conjunto de metas de cara a su agenda para el 2030, dentro de los que se insta a los distintos gobiernos del mundo a trabajar por la gente y para la gente, garantizando elevar los niveles de resiliencia humana, cuyo propósito se ha de lograr en un esfuerzo escalonado que comprende ejecución, aprendizaje, implantación y documentación de las lecciones aprendidas en el proceso.

Dentro de los objetivos de desarrollo del milenio, ODM, las personas representan un nivel de trabajo macro, dada las implicaciones que tiene para un Estado, políticamente hablando, sus habitantes, tanto humanos como la flora y fauna, y por lo tanto se ha establecido dentro de los trabajos el crear mejores condiciones de vida y que a su vez aboguen por la conservación medio ambiental. El acceso a la energía es uno de los ODM más dinámicos que existen tal y como se observará a continuación.

Para la República Dominicana es de vital importancia el lograr un equilibrio entre el empleo de los recursos estatales para con el desarrollo y crecimiento de las personas. La investigación realizada aporta datos relevantes sobre la condición del acceso a la energía en el país dominicano y también la calidad del servicio, así como su implicación en la vida cotidiana de las personas.

1. Es necesario que el gobierno desarrolle políticas que garanticen la continuidad de los planes a largo plazo, dado que, según los datos e impresiones recogidas, esta es una debilidad que dificulta el logro de los objetivos 
de crecimiento y sostenibilidad. Una normativa de continuidad no sólo mermaría el cierre de proyectos, sino que también ayudaría a disminuir el grado de incertidumbre de los inversionistas privados, ya sean estos locales o extranjeros.

2. Las políticas hacia el desarrollo y crecimiento de las personas y sus comunidades es otro punto que refleja una falta de enfoque, al menos uno objetivo y eficiente, pues las opiniones recopiladas entre los distintos encuestados denota que si bien se ha logrado algún nivel de mejoría en la última década, no es menos a lugar que la centralización de las decisiones no han favorecido a una verdadera revolución en la que la gente pueda tener iniciativas comerciales, sociales o culturales que les permitan crecer de forma individual y colectiva.

\section{La energía como bien y servicio a} las personas no tiene los niveles de accesibilidad deseados de cara a la agenda del milenio. A pesar de encontrar una red de electrificación que cubre la mayor parte del territorio nacional, la oferta del mismo tiene un complicado esquema de adquisición, siendo notable que aún existen zonas en donde falta electrificar $y$, pero para otras en donde a pesar de contar con el tendido y la prestación, ésta es muy deficiente o casi inexistente; se encontró por ejemplo zonas en donde hay quince días de apagones.

4. La energía como insumo productivo carece de unos niveles de eficiencia y efectividad que permitan a los productores y comerciantes emprender nuevos negocios, en casos más extremos lograr mantener los existentes. La problemática principal se debe a los precios altos de la energía en el país, lo cual viene dado por el mismo esquema de producción, ello porque el Estado tiene que comprar a terceros para revender.

5. Los precios del petróleo son un factor decisivo para lo que es la transformación de las distintas fuentes de producción energética de dependen de la cotización del mismo, pues si bien se tiene acuerdos comerciales con países como Venezuela, la variable que más afecta es la tasa del dólar, dado que la compra se realiza en esa moneda. En su conjunto, estas dos últimas condicionan la comercialización final de la electricidad $y$, consecuentemente termina en un incremente de costos para las distintas organizaciones, instituciones, productores independientes, entre otros. Al final, la desventaja se convierte en una factura más cara para el consumidor final, pero también una carga para una gran cantidad de bienes y servicios de uso diario.

6. Los niveles de pobreza se pueden asociar con el acceso a los servicios básicos, siendo la energía uno de los más relevantes en los tiempos actuales. La investigación aporta evidencias de que tener el servicio eléctrico influye en no sólo en la calidad de vida de la gente, sino que también se relaciona con otros aspectos como la salud, una mejor culturización y convivencia social, así como también mayor grado de información cotidiana, tanto a nivel local como internacional. Lo anterior sucede porque se puede tener mejor refrigeración, uso de computa- 
doras, televisiones, etc. Los noticieros ayudan a mantener cierto grado de actualización del acontecer diario.

7. La percepción del gobierno que tienen las personas afectarán en cierto grado la receptividad hacia el encaminamiento de nuevos proyectos de energía renovable, dado que los datos recogidos dejan ver que la confiabilidad es baja cuando se trata de invertir los recursos del pueblo, por lo que el grado de resistencia al cambio se mantiene aun conociendo algunos de los beneficios de este tipo de proyectos.

8. El aprovechamiento de los recursos naturales como fuentes de energía sostenible requiere de una mayor inversión, además de un programa de incentivos y exenciones fiscales que potencien la masificación de proyectos de energía renovable a partir de la abundancia de cuencas hidrográficas, sol, viento y aguas oceánicas.

9. El gobierno puede dentro de su estrategia de sostenibilidad usar la dotación de energía en las zonas rurales para incentivar a la permanencia de las personas en el campo y de tal forma continuar en las labores agrícolas, con lo que se beneficiaría la comunidad al tener bienes de consumo de primera necesidad más asequibles y a mejores precios. Pero además combatiría la emigración hacia las grandes ciudades y por ende la sobrepoblación de las mismas, con lo que se encaminaría al cumplimiento de uno de los objetivos de desarrollo del milenio de la ONU, que es lograr la resiliencia de las personas y mejorar los asentamientos humanos.
10. La masificación de la producción energética a partir de fuentes renovables tendría un impacto también en el transporte metropolitano, al poder dotar a las grandes ciudades de sistemas modernos y amigables con el medio ambiente, logrando por tanto un descongestionamiento del tráfico vial, uno de los problemas que han persistido en el país dominicano a través de las décadas.

\section{Recomendaciones:}

a. Se recomienda al gobierno dominicano crear los mecanismos que garanticen la continuidad de la planificación a largo plazo en el territorio nacional, así como fortalecer la cooperación internacional en materia de ejecución e implementación de proyectos de renovación tecnológica. Si bien es cierto que el marco normativo cuenta con leyes y resoluciones que delimitan lo que debe hacerse, no se incluye el cómo de forma tal que las autoridades consecuentemente sigan el curso de las acciones previstas.

b. En materia de transparencia se debe mejorar el esquema de licitaciones y contrataciones de las constructoras de plantas de generación eléctrica. En la actualidad el país está dentro de los muchos que recibieron sobornos por parte de la empresa Odebrecht para ser favorecida esta última con la concesión de proyectos gigantes, tal como el caso de Punta Catalina, el cual fue incluso auditado, pero aún persisten las dudas en la población sobre la autenticidad de la misma.

c. Trabajar con la educación de las personas a nivel nacional debe for- 
mar parte crucial del plan de mejoramiento de la estructura de la oferta del servicio energético, pero además crear conciencia en la gente de la importancia del ahorro de energía y sus consecuencias en el cambio climático, dado que República Dominicana es uno de los países que más sufrirán los efectos del fenómeno en las décadas futuras, tal como se evidencia en la investigación y los propios datos de la ONU.

d. Mejorar la política de incentivos y exenciones fiscales sobre los proyectos de energía renovables, así como también con otro tipo de implementación y renovación tecnológica que beneficie a la conservación del medio ambiente, reduciendo en consecuencia la huella de carbono. En este particular se destaca la Ley 103-13 de incentivo a la importación de vehículos de energía no convencional, misma que puede servir de modelo para otras áreas de la macro economía, favoreciendo la producción verde y limpia.

e. Masificar la construcción de presas e implementar un sistema de acumulación de aguas lluvias, con lo que se lograría primero medir la cantidad de recursos pluviales, con la consecuencia segunda de poder destinar estas como abastecimiento para la producción agrícola, agua potable, entre otros.

f. Continuar la expansión de los campos eólicos, como el proyecto Los Cocos y Larimar, dado que el país posee las características ambientales y geográficas que favorecen este tipo de plantas de producción; sobre todo en las áreas costeras, dentro de las cuales se encuentran 16 de las 32 provincias, incluida la capital.

g. Motivar a las empresas privadas a cooperar con el Estado en la creación de proyectos de energía solar, los cuales se pueden masificar a manera unifamiliar y también como de expansión provincial, es decir, que para las áreas remotas en donde la electrificación por redes no es factible por su distancia o carencia de presupuesto, se pueden emplear paneles solares en las viviendas.

h. Limitar o reducir la dependencia del uso de combustibles fósiles para la producción energética en general, pues si bien se han tomado decisiones en este ámbito, el plan debe ser más agresivo, incentivando más al uso de la biomasa, por ejemplo, en sustitución del carbón, gasoil y gasolina.

i. Pactar con los productores nacionales para lograr una producción de bio combustibles a gran escala y de tal forma abastecer a las plantas generadoras de electricidad a un nivel escalado que termine con la emisión masiva de dióxido de carbono.

j. Para las investigaciones futuras que tomen como referencia los resultados encontrados en el trabajo desarrollado, tener en cuenta que se deberá ampliar el horizonte temporal para poder reajustar las proyecciones realizadas a la población con respecto al consumo energético.

k. Futuras investigaciones pueden utilizar los datos y hallazgos obtenidos para ampliar hacia otras el marco de aplicación a otras provincias del país dominicano y de tal 
forma contrastarlos a la realidad de cada región local, con lo que se podría abordar la problemática con distintas estrategias.

I. La metodología utilizada podrá ser replicada siempre que se tomen parámetros similares para la selección de muestras cualitativas, entendiendo que integran de forma correcta a distintos estratos de la sociedad dominicana y, sería útil en otros países con buenos resultados.

\section{Referencias}

Bolkesjø, T., Eltvig, P., Nygaard, E. (2014). An Econometric Analysis of Supportscheme Effects on Renewable Energy Investments in Europe.Procedia 58 2 - 8. Recuperado de: http://www. sciencedirect.com/science/article/pii/ S1876610214017688

Botelho, A., Pinto, L., Lourenço, L., Valente, M., Sousa, S. (2016). Public perceptions of environmental friendliness of renewable energy power plants. Energy Procedia 106 73-86. Recuperado de: https:// repositorium.sdum.uminho.pt/bitstream/1822/44041/1/10.1016\%3Aj. egy pro.2016.12.106.pdf

Bossink, B., (2017). Demonstrating sustainable energy: A review based model of sustainable energy demonstration projects. Renewable and Sustainable Energy Reviews. Recuperado de: http://dx. doi.org/10.1016/j.rser.2017.02.002

Corporación Dominicana de Empresas Eléctricas Estatales, CDEEE (2013). Plan Estratégico 2013-16. Recuperado de: http://cdeee.gob.do/cdeeesite/?wpfb_dl=131

Ecodiseño. (2011). Eco innovación. Tres casos de éxito. Recuperado de: http:// www.xn-eco-diseo-s6a.net/script/ photo/1353072639aido\%20ecoinnovacion.pdf

Economipedia. (2015). Índice de Gini. Recuperado de: http://economipedia. com/definiciones/indice-de-gini.html

Foster, E., Contestabile, M., Blazquez, J., Manzano, B., Workmana, M., Shah, N. (2017). The unstudied barriers to widespread renewable energy deployment: Fossil fuel price responses. Energy Policy 103 258-264. Recuperado de: http://www.sciencedirect.com/science/article/pii/S0301421516307157

Gallardo Lichaá, N., \& Franco, J. (2017). Proceso de Mentoring en la innovación disruptiva de las organizaciones. Revista GEON (Gestión, Organizaciones Y Negocios), 4(1), 80-90. https:// doi.org/10.22579/23463910.46

Global Wind Energy Council. (2013). 30 years of policies for wind energy. Irena.Recuperado de: www.gwec.net/publications

Greenpeace. (2014). El impacto de las energías renovables en los hogares. Recuperado de: http://www.greenpeace.org/espana/Global/espana/2014/ Report/cambio- climatico/Informe\%20 ER\%20Hogares.pdf

Grupo de trabajo III del Grupo Intergubernamental de Expertos sobre el Cambio Climático, IPCC. (2011). Fuentes de energía renovables y mitigación del cambio climático: resumen para responsables de políticas y resumen técnico. Recuperado de: http://www. ipcc.ch/pdf/special-reports/srren/SRREN_FD_SPM_final.pdf

Hernández, R., Fernández, C. y M. Sampieri. (2010). Metodología de la investigación. 
México. McGraw-Hill / Interamericana Editores, S.A. Quinta Edición. ISBN: 978-607-15-0291-9

Ibrahiem, D. (2015). Renewable electricity consumption, foreign direct investment and economic growth in Egypt: An ARDL approach. Procedia Economics and Finance 30313-323. Recuperado de: https://www.researchgate.net/ publication/284114336_Renewable_ Electricity_C onsumption_Foreign_Direct_Investment_and_Economic_ Growth_in_Egypt_An_ARDL_Approach

International Energy Agency. IEA. (2015). Indicadores de Eficiencia Energética: Bases esenciales para el establecimiento de políticas. Recuperado de: https://www.iea.org/publications/freepublications/publication/ EnergyEfficiency Vespagnol_epdf.pdf

Karatayev, M., Clarke, M. (2014). Current energy resources in Kazakhstan and the potential of renewables: A review. Energy Procedia 59 - 104. Recuperado de: http://www.sciencedirect.com/science/article/pii/S1876610214017214

Lindhult, E., Campillo, J., Dahlquist, E., Read, S. (2015). Innovation capabilities and challenges for energy smart development in medium sized European cities. Energy Procedia 88205 - 211. Recuperado de: http://www. sciencedirect.com/science/article/pii/ S1876610216301217

Listín Diario. (2017). Economía: CDEEE anuncia inversión de US\$780 millones en ocho proyectos de energía renovable. Recuperado de: http://www.listindiario.com/economia/2017/04/04/460737/cdeee-anuncia-inversion-de-us-780-millones-enocho-proyectos-de-energia-renovable

Organization for Economic Cooperation and Development, OECD. (2015). En- vironment at a Glance 2015: OECD Indicators. OECD Publishing.

Paris. Recuperado de: http://www. oecd-ilibrary.org/environment/ environment-at-a-glance_19964064 DOl:http://dx.doi.org/10.1787/9 789264235199-en

Oficina Nacional de Estadística, ONE. (2016). Estimaciones y proyecciones nacionales de población 19502100. Recuperado de: https://www. one.gob.do/Estadisticas/8/proyecciones-de-poblacion

Organismo Internacional de Energía Atómica, OIEA. (2008). Indicadores energéticos del desarrollo sostenible: Directrices y metodologías. Recuperado de: http://www- pub.iaea.org/MTCD/ publications/PDF/Pub1222s_web.pdf

Organización de las Naciones Unidas, ONU. (1992). Convención marco de las Naciones Unidas sobre el cambio Climático. Recuperado de: http://unfccc. int/resource/docs/convkp/convsp.pdf (S.F.). Energía para el desarrollo sostenible. Desarrollo sostenible: Plataforma de conocimiento.

Osunmuyiwaa, O., Kalfagianni, A. (2017). Transitions in unlikely places: Exploring the conditions for renewable energy adoption in Nigeria. Environmental Innovation and Societal Transitions 22 26-40. Recuperado de: http://www. sciencedirect.com/science/article/pii/ S221042241630048X

Quintana, D., Díaz, O., Salinas, G., Casas, M., Huitrón, J., Beltrán, R., Guerrero, E. (2011). Desarrollo sustentable en el contexto actual. Educar para la sustentabilidad: paradigma de cambio $y$ conservación. Cop 15. ISBN: 978-14583-7188-1 Recuperado de: http:// campusvirtual.ucimexico.edu.mx/ $\mathrm{mod} / \mathrm{resource} /$ view.php? $\mathrm{id}=26389$ 
Sari, A., Akkaya, M. (2016). Contribution of Renewable Energy Potential to Sustainable Employment. Procedia - Social and Behavioral Sciences 229 316-325. Recuperado de: https://www.researchgate.net/publication/307999492_ Contribution_of_Renewab le_Energy_ Potential_to_Sustainable_Employment

Tantau, A., Chinie, A., Carlea, C. (2015). Corporate Entrepreneurship and Innovation in the Renewable Energy Field. Procedia Economics and Finance 22 353 - 362. Recuperado de: http:// www.sciencedirect.com/science/article/pii/S2212567115003020

Silva, G., Di Serio, L. (2016). The sixth wave of innovation: are we ready? RAI Revista de Administração e Inovação 13 (2016) 128-134. Brasil. Recuperado de: http://www.revistas.usp.br/rai/article/view/103493/114212

Shukla, A., Sudhakar, K., Baredar, P. (2017). Renewable energy resources in South Asian countries: Challenges, policy and recommendations. Resource-Efficient Technologies. Recuperado de: http:// www.sciencedirect.com/science/article/pii/S2405653716302299

Sosa, M., Hernández, F. y Fuente, F. (2007). Desarrollo sostenible: sus dimensiones. El Cid Editor. ISBN: 10257007. Recuperado de: http:// site.ebrary.com/lib/eudesp/reader.action?doclD=10159956\&ppg=11

Sustainable energy for all, SE4ALL. (2015). Progress toward sustainable energy. Global tracking framework 2015 key findings. Recuperado de: https:// www.iea.org/media/news/2015/news/ GlobalTrackingFramework2015Ke yFindings.pdf

United Nations. (2014). Synthesis of energy-related issues highlighted in national reports of Rio+20. Division for Sustainable Development. United Nations Department of Economic and Social Affairs. Recuperado de: https://sustainabledevelopment.un.org/content/ documents/1258Energy\%20Docu ment.pdf (2014). Partnerships Briefs for Small Island Developing States.

Sustainable Energy. Recuperado de: http://www.sids2014.org/content/ documents/3481354SIDS_BRIEFS_SocialDvp mt.pdf

United Nations Environment Programme. UNEP. (2014). Eco Innovation manual: working version for a pilot application. Recuperado de: https://drive.google. com/drive/u/0/folders/0B5reveARoVTfb18ybDFobHRvbU0

(2011). Environment and energy: energy for people-centred sustainable development. Recuperado de: https://sustainabledevelopment.un.org/content/ documents/957055_Energy\%20fo r\%20People\%20Centred\%20Sust\%20 Dev\%202011_brochure.pdf

Xercavins, J., Cayuela, D., Cervantes, G. (2005). Desarrollo sostenible. Catalunya. España. Edicions UPC. Recuperado de: http://site.ebrary.com/lib/eudesp/ reader. action?docID=11046366\&ppg $=83$ 